GONZÁLEZ GUERRERO, P. -1927- Contribución al estudio de las algas y Esquizófitas de España. Trabajos del Museo Nacional de Ciencias Naturales. Serie Botánica, 22:1-52. GONZÁlEZ GUERRERO, P. -1929- Nuevos datos de plancton hispano-marroquí (agua dulce). Bol. R. Soc. Esp. Hist. Nat., 29:251-254.

GONZÁLEZ GUERRERO, P. -1965- Las algas de la sílice. Anals. Inst. Bot. A.J. Cavanilles, XXIII: $107-143$

GREUTER, W. (ed.). -1988-International Code of Botanical Nomenclature. XIV International Botanical Congress. Berlín 1987. Cramer.

KOL, E. -1928- Ueber die Kryovegetation der Hohen-Tátra. I. Folia Cryptog., 1:614-622.

VAVRA, J. \& J. VAVRA -1968- In memoriam Marssoniella Lemm. 1900. Arch. Protistenk., 3:12-17.

MARVANOVA, L., P. MARVAN, \& J. RUZICKA -1967- Gyoerffyella Kol. 1928, a genus of the hyphomycetes. Persoonia, 5(1):29-44.

PRINTZ, H. -1914- Kristianatraktens Protococcoideer. Skrifter udgat Videnskabsselskabet $i$ Christiania Mathematik-Natur. KL, 6:1-123.

RANZONI, F.V. 1-953- The aquatic hyphomycetes of California. Farlowia, 4:353-398.

PRINTZ, P.F. -1888- Familiae Polyedriarum Monographia. Notarisia, 3:493-516.

TUBAKI, K. -1958- Studies on Japanese hyphomycetes V. Leaf and stem group with a discussion of the classification of hyphomycetes and their perfect stages. J. Hatt. Bot. Lab., 20:142-244.

(Aceptado para su publicación en junio de 1991)

Dirección de los autores: A. Roldán: Unidad de recursos naturales. CEBAS-CSIC. Apdo. 4195. E-30080 Murcia. M. Aboal: Departamento de Biología Vegetal (Botánica). Facultad de Biología. Universidad de Murcia. E-30100. Murcia.

\title{
CONTRIBUCIÓN A LA COROLOGÍA DE LOS BRIÓFITOS DE GALICIA
}

Palabras clave. Briófitos, Galicia.

\section{Juan REINOSO FRANCO y $\mathbf{M}^{\mathrm{a}}$ del Carmen VIERA BENÍTEZ}

Continuando con nuestros estudios acerca de la flora briológica gallega, reseñamos en esta nota la presencia en la región de 6 nuevas especies y se confirman otras 8 citadas anteriormente en la bibliografía que constituyen novedad para la provincia de Lugo. Las nuevas citas para Galicia se señalan con un asterisco $(*)$.

Brachythecium velutinum (Hedw.) B.S.G.

Orbazay (Lugo) 29TPH1563. Sobre roca granítica. (SANT-bryo. 613). En Galicia sólo se conocía la referencia de Reinoso (1984) para la provincia de La Coruña, constituye, por lo tanto, primera cita para la provincia de Lugo. 
Bryum flaccidum Brid.

Orbazay (Lugo) 29TPH1563. Sobre roca granítica. (SANT-bryo. 750). Novedad para la provincia de Lugo y segunda cita regional pues sólo se conoce la inicial referencia de Reinoso (1984) que señala el taxon en La Coruña.

Bryum pseudotriquetrum (Hedw.) Gaertn. Meyer \& Schreb.

Vilachá de Mera (Lugo) 29TPH961. Sobre roca granítica. (SANT-bryo. 765). Novedad para la provincia de Lugo.

(*) Bryum uliginosum (Brid.) B.S.G.

Santiago de Compostela 29TNJ3847. Sobre un muro de contención. (SANT-bryo. 766).

Cynodontium bruntonii (Sm.) B.S.G.

Vilachá de Mera (Lugo) 29TPH961. Epífita de Quercus pyrenaica (SANT-bryo. 915). Astariz (Lugo) 29TPH361. Sobre roca granítica. (SANT-bryo. 916). Las únicas referencias gallegas corresponden a Colmeiro (1867), Casares (1915) y Reinoso (1984). Primera cita para la provincia lucense.

Dicranodontium denudatum (Brid.) Britt.

Vilachá de Mera (Lugo) 29TPH0961. Epífita de Quercus robur. (SANT-bryo. 1059). Reseñada en la provincia de La Coruña por Reinoso y Alvarez (1984), a la que hay que añadir la inicial referencia de Merino (1916) que señala el taxon en La Guardia (Pontevedra). Novedad para la provincia de Lugo.

Fissidens viridulus (Sm.) Wahlenb.

Vilachá de Mera (Lugo) 29TPH09061. Sobre roca granítica. (SANT-bryo. 1160). Se amplía el área de distribución de esta especie de la que se conocían referencias anteriores de Merino (1916), Luisier (1918) y Reinoso (1984). Novedad para la provincia de Lugo.

Grimmia trichophylla Grev.

Bacurín (Lugo) 29TPH0659. Sobre muro de contención. (SANT-bryo. 1314). Casares (1915) refiere una cita de Colmeiro para Orense; Reinoso y Alvarez (1984) señalan el taxon en Santiago. Novedad para la provincia de Lugo.

(*) Mnium thomsonii Schimp.

Veral (Lugo) 29TPH1261. Epífito de Alnus glutinosa. (SANT-bryo. 1715)

(*) Orthotrichum anomalum Hedw.

Bacurín (Lugo) 29TPH659. Sobre granitos. (SANT-bryo. 1812).

(*) Scapania irrigua (Ness.) Gott. et al.

Veral (Lugo) 29TPH1261. Epífita de Alnus glutinosa. (SANT-bryo. 2231). Orbazay (Lugo) 29TPH1563. Sobre granitos. (SANT-bryo. 2232).

(*) Tortella tortuosa (Hedw.) Brid.

San Pedro de Mera (Lugo) 29TPH0957. Sobre Granitos. (SANT-bryo. 2316). 
(*) Tortula laevipila (Brid.) Schwaegr. var. meridionalis (Schimp.) Wijk.

Santiago de Compostela 29TNJ3847. Epífita de Salix sp. (SANT-bryo. 2412).

Tortula papillosa Wills.

Santiago de Compostela 29TNJ3847. Epífita de Robinia Pseudoacacia. (SANT-bryo. 2426). Se confirma la presencia de esta especie en Galicia de la que se conocía una única cita debida a Casares (1915). Novedad para la provincia de Lugo.

\section{BIBLIOGRAFÍA}

CASARES-GIL, A. -1915- Enumeración y distribución de las Muscíneas de la Península Ibérica. Trab. Mus. Nac. Cien. Nat. Ser. Bot., 8:1-179. Madrid.

COLMEIRO, M. -1867-Enumeración de las Criptógamas de España y Portugal. Rev. Progresos Cienc., 16-17:54-519. Madrid.

LUSIER, A. -1918- Fragments de Bryologie Ibérique. 14. Mousses de Galice. Broteria Ser. Bot, 16:123-142. Braga.

MERINO, P. -1916- Contribución a la Muscología de la Península Ibérica. Bol. R. Soc. Esp. Hist. Nat., 16:270-276. Madrid.

POTIER DE LA VARDE, R. -1945- Liste des espèces du Genre Fissidens récoltées dans la Péninsule Ibérique par M. et Mme. Allorge. Rev. Bryol. Lichénol, 15:30-39. Paris.

REINOSO, J. -1984- Contribución al conocimiento de la flora briológica de Galicia. Briófitos de la fraga de Caaveiro (La Coruña). I. Musgos. Lazaroa, 6:237-247. Madrid.

REINOSO, J. y J. ÁLVAREZ - 1984- Estudio briológico de la cuenca del río de Rojos (Santiago). Acta Científica Compostelana, 21:273-284. Santiago de Compostela.

(Aceptado para su publicación en enero de 1991)

Dirección de los autores: Departamento de Biología Vegetal (Botánica). Facultad de Biología. Universidad de Santiago. 15706. Santiago de Compostela.

\section{NUEVA LOCALIDAD DE ASTRAGALUS NITIDIFLORUS JIMÉNEZ Y PAU EN ESPAÑA}

Palabras clave: Corología, Astragalus, Fabaceae.

Francisco M. VÁZQUEZ, Eugenio DOMÍNGUEZ y Juan Antonio DEVESA

Al estudiar el género Astragalus en la región extremeña se han detectado unas poblaciones en el $\mathrm{S}$ de la provincia de Badajoz cuya identificación no resulta fácil mediante las floras al uso.

Siguiendo la monografía de Bunge (1868 \& 1869) sobre Astragalus, y considerando el carácter anual de nuestras plantas, los individuos pacenses pertenecen 